Relations industrielles

Industrial Relations

\title{
The French Labor Courts : Judgement by Peers, par William H. MacPherson et Frederic Meyers, Institute of Labor and Industrial Relations, University of Illinois, Urbana, 1966, 104 pages.
}

\section{Hubert Reid}

Volume 22, numéro 4, 1967

URI : https://id.erudit.org/iderudit/027858ar

DOI : https://doi.org/10.7202/027858ar

Aller au sommaire du numéro

Éditeur(s)

Département des relations industrielles de l'Université Laval

ISSN

0034-379X (imprimé)

1703-8138 (numérique)

Découvrir la revue

Citer ce compte rendu

Reid, H. (1967). Compte rendu de [The French Labor Courts : Judgement by Peers, par William H. MacPherson et Frederic Meyers, Institute of Labor and Industrial Relations, University of Illinois, Urbana, 1966, 104 pages.] Relations industrielles / Industrial Relations, 22(4), 591-592.

https://doi.org/10.7202/027858ar

Tous droits réservés @ C Département des relations industrielles de l'Universite Laval, 1967
Ce document est protégé par la loi sur le droit d'auteur. L'utilisation des services d'Érudit (y compris la reproduction) est assujettie à sa politique d'utilisation que vous pouvez consulter en ligne.

https://apropos.erudit.org/fr/usagers/politique-dutilisation/ 
Le grand avantage de ce volume est de présenter un éventail des nombreuses sources de la psychologie industrielle ainsi que les contributions des principoux chercheurs dans ce domaine. II s'agit d'un volume très intéressont pour celui qui veut explorer de façon générale la psychologie industrielle et très utile sur le plan académique pour quiconque veut confronter les diverses théories en cours

\section{Jean BOIVIN}

\section{Rapport de la Commission Royale sur les Relations Employeurs-Employés dans les Services Publics du Nouveau-Brunswick, por Dr Soul J. Frankel, 1967, 111 pages}

Ce ropport nous présente l'étude menée par la Commission sur trois champs et en trois mouvements, tels qu'arrêtés dans la décret en conseil 66-496, du 29 juin 1966.

1) Etude d'abord des relations employeursemployés dans le Service Civil;

2) L'enseignement;

3) Les hôpitaux.

Le rapport étudie brièvement les tendances actuelles dans chacun des secteurs, les mémoires qui furent présentés d̀ la commission et les recommandations de cette dernière

En fait, la distinction des trois secteurs (Service Civil, Commissions Scolaires, Hôpitoux) s'otténuent énormément sous l'aspect des relations employeurs-employés quand le ropport signale la prise de responsabilité presque totale du financement de l'enseionement et des services hospitaliers. II s'agit donc de trois groupes d'employés du gouvernement.

Lo Commission d'enquête tient compte de cette situation, et propose sensiblement les mêmes législations et politiques oux relations entre employeurs et employés touchant lo syndicalisation (unités de négociation), la négociation (procédure), la convention collective. Elle tient compte cependant de l'évolution et de l'organisation de ces trois secteurs selon la législation et les circonstances particulières de l'organisation professionnelle de ces groupes.

Le rapport décrit les principaux problemes de la syndicalisation du secteur public et désigne les forces en présence. Le problème est posé quant à l'ancienne législation (Loi des Relations du Travail), aux organismes para-syndicaux et associations professionnelles déjò existantes, oux diffé- rents employeurs: Etat, Corporations et Commissions de la Couronne, Association Hospitalière et Association des Commissaires d'Ecoles

Enfin, tout un processus de négociotion ollant de la discussion permanente d lo grève est défini, de même que la distinction des unités de négociation, selon les caractères communs et distincts des différentes cotégories professionnelles.

En annexe sont ajoutées d'excellentes statistiques qui décrivent l'élargissement des chomps d'action de l'Etat et la croissance des effectifs de la main-d'oeurre employés dans le secteur public

\section{L.-René PARENTEAU}

\section{The French Labor Courts: Judgment by}

Peers, par William H. MacPherson et Frederic Meyers, Institute of Lobor and Industrial Relations, University of Illinois, Urbana, 1966, 104 pages

Ayant constaté à plusieurs reprises que les méthodes utilisées en Europe pour régler les conflits de travail semblaient fort méconnues aux Etats-Unis, MacPherson et Meyers ont décidé d'effectuer une étude empirique sur le fonctionnement des conseils de prud'hommes, en France. Et, par souci d'objectivité, ils se sont limités à une analyse de cette institution plutôt qu'à la recherche d'une hypothétique supériorité d'un des systèmes sur l'autre.

Dans un premier chapitre consacré à l'introduction, les auteurs ont établi les objectifs qu'ils s'étaient fixés en entreprenont cette étude. Ils ont de plus précisé d̀ leurs lecteurs américains deux différences fondamentales entre le système de leur pays et celui qu'ils ont étudié:

1) le nombre d'avantoges sociaux dont bénéficie le travailleur français en vertu de la loi, alors que l'ouvrier américain doit encore, actuellement, en négocier en très grand nombre.

2) La présence exclusive, comme juges, de non juristes élus par les employeurs et les employés.

Après un deuxième chapitre relatant I'histoire des conseils de prud'hommes - vieille de plus de cent cinquante ans - les auteurs étudient, dans le troisième, lo structure, la compétence et la procédure actuelles de ces tribunaux. On doit noter qu'il existe actuellement environ deux cent cinquante 
conseils de prud'hommes bien structurés, chacun d'eux pouvant comporter quatre sections spécialisées (industrie, commerce, agriculture et divers). De plus, dans les centres principaux, la section industrie se subdivise en quatre sous-sections (chimie, construction, métal et textile)

Le chapitre suivant présente les résultats d'une recherche effectuée auprès des conseils de prud'hommes parisiens. Les auteurs se sont efforcés de décrire ici le fonctionnement réel de ces tribunaux, en expliquant le rôle propre du «Bureau spécial », dont lc tâche principale est de rechercher la conciliation et du « Bureau Général » qui fait fonction de tribunal lorsque la séance de conciliation $n^{\prime} a$ pas produit des résultats sérieux.

Dans le cinquième chapitre, MacPherson et Meyers ont présenté une analyse des données qu'ils avoient obtenues en définissant les problèmes auxquels ces juridictions sont confrontées (bipartisme, système électoral, etc.)

Le sixième chapitre constitue une espèce de bilan de l'Institution alors que le septiè- me comporte les conclusions principales des outeurs

Ces conclusions concernent principalement le bipartisme de ces tribunaux, la procédure d'élection de leurs juges, la composition des tribunaux susceptibles d'entendre l'appel des décisions des tribunaux inférieurs et le rôle joué par les cours ordinaires en cos d'inexistonce de conseils de prud'hommes dans une région

A notre avis, les auteurs font réellement vivre sous nos yeux les tribunaux spécialisés et en présentent les avantages et inconvénients de façon apparemment fort objective II est toutefois permis de se demander si lo réalité parisienne est typique de celle de la France entière; on peut en douter. Ainsi, par exemple, il est peu probable que MocPherson et Meyers aient vanté avec autant de conviction l'objectivité des juges, s'ils avaient analysé le comportement de ceux d'une petite municipalité bretonne Cette extrapolation de leur part n'infléchit pas la valeur de leurs observations mais soulève tcutefois un certain scepticisme chez le lecteur quant à son bien-fondé.

Hubert REID

\section{PUBLICATIONS RECENTES}

\section{GÉNÉRALITÉS}

\&Population Explosion the Standard of Living and Social Conflict», by J.E. Meade, The Economic Journal, Vol. LXXVII, No 306, June 1967, pp. 233-256.

«Approaches to Educational Planning 》, by $M$. Blaug, The Economic Journal, Vol LXXVII, No. 306, June 1967, pp. 262-288.

\& The Production of Human Capital and the Life Cycle of Earnings », by Yoram BenPorath, The Journal of Political Economy, Vol. 75, No. 4, August 1967, part 1, pp. $352-366$

«Economic Security and Social Conflict in America: The Early Twentieth Century, Part I 》, by Roy Lubove, Journal of Social History, Vol. 1, No 1, Fall 1967, pp. 61-88

«Le changement social », par René et Henri Théry, Recherche Sociale, no 11, mai-juin $1967,44 \mathrm{pp}$
«Planificateurs et planification aux EtatsUnis», par Charles Roig, l'Actualité économique, $43 \mathrm{e}$ année, no 2, juillet-septembre 1967, pp. 280-338.

«Les méthodes de lo planification régionale en U.R.S.S. », par Ch. Beaucourt, Economies et sociétés, Série G-25, mars 1967, pp. 199.215.

«Les classes sociales au Canada», por Alfred Dubuc, Annales, $22 e$ année, no 4 , juillet-août 1967, pp. $829-845$.

\& Pauvres et pauvreté dans les sociétés riches », Economie et Humanisme, no 174 mai-juin 1967, 94 pp

«Administration, frein ou moteur du développement 》, Dévoloppement et Civilisations, no 29 , mars 1967, $114 \mathrm{pp}$

«La planification: rôle et limites de son intervention », par $M$. Ripert, Humanisme et Entreprise, no 44, août 1967, pp. 65-80. 